\title{
¿Es la diabetes tipo 2 una enfermedad tratable quirúrgicamente?
} Revisión de una hipótesis

Romero-lbargüengoitia $\mathrm{ME}^{*}$

\section{- Resumen}

- La diabetes tipo 2 es una enfermedad de creciente desarrollo epidemiológico en México y en el mundo, por lo que es prioritario el mejor entendimiento de dicha enfermedad y la investigación de nuevas opciones

- terapéuticas encaminadas a un mejor control de la en-

- fermedad.Además de la importante reducción de peso

- de los pacientes sometidos a cirugía bariátrica, existe

- evidencia de la restauración de los niveles de glucosa y

- cifras de hemoglobina A lc aún con la descontinuación

del tratamiento médico antes de que el paciente pierda peso, lo cual sugiere que existen otros procesos asociados a la modificación de la anatomía gastrointestinal, lo que produce cambios en la secreción de los péptidos intestinales que forman el eje enteroinsular, que podrían explicar dicha mejoría. Este artículo presenta una revisión de dichos cambios para valorar si se puede o no considerar a la diabetes como una enfermedad tratable quirúrgicamente. LUXMEDICA 2009; 4(II):29-35.

Palabras clave: diabetes tipo 2, cirugía bariátrica, eje entero-insular.

\section{Introducción}

La diabetes tipo 2 es una enfermedad de gran importancia epidemiológica, con una incidencia creciente a nivel mundial de $37 \%{ }^{1}$. En la población de México existe una prevalencia reportada de $10.7 \%^{2}$. Es prioritario un mayor entendimiento de dicha enfermedad mediante la investigación de nuevas opciones terapéuticas encaminadas al mejor control de la enfermedad.

Existe evidencia de la restauración de los niveles de glucosa y cifras de hemoglobina $A 1 C$ en pacientes sometidos a cirugía ba-

\footnotetext{
* Universidad Autónoma de Aguascalientes. Instituto de Ciencias Médicas y Nutrición Salvador Zubirán
} 
riátrica ${ }^{3-8}$. La normalización sin tratamiento se ha observado antes de que el paciente pierda peso ${ }^{9}$, lo cual sugiere que existen otros procesos asociados a la modificación de la anatomía gastrointestinal que podrían explicar dicha mejoría.

Aunque no se conocen exactamente los mecanismos moleculares de lo anteriormente comentado, el objetivo de la siguiente revisión es exponer la evidencia que existe al respecto y valorar si es factible o no considerar a la diabetes como una enfermedad que puede ser controlada quirúrgicamente.

\section{| | | | | | | | | | | | | | | | | | | | | | | | | | | | | | | | | | | | | | | | | | | | | | | | | | | | | | | | | | | | | | | | | | | | | | | | | | | | | | | | | | | | | | | | | | | | | | | | | | | | | | | | ||}

\section{Diabetes 2 como una enfermedad intestinal}

Existen estudios desde 1902 donde se describe la existencia de factores producidos por la mucosa gástrica que se llamaban "secretinas" con importante papel en el funcionamiento endócrino del páncreas ${ }^{10}$.

Hoy en día dicha relación se explica a través del "eje enteroinsular" formado por las incretinas péptido-1 similar al glucagón (GLP-1) y péptido insulinotrópico dependiente de glucosa (GIP), además del péptido YY (PYY), grelina, colecistoquinina (CCK) y polipéptido pancreático (PP). El GLP-1, producido por las células $L$ del íleo distal, participan en la potencialización de la secreción de la insulina mediada por glucosa ${ }^{11}$, inhibe la secreción de glucagón, disminuye el vaciamiento gástrico, induce saciedad, disminuye el consumo de alimento, incrementa la lipogénesis ${ }^{12} \mathrm{e}$ incrementa la masa de las células B pancreáticas a través de procesos de regulación, proliferación, neogénesis $y$ apoptosis ${ }^{13}$. El GIP, producido por las células $\mathrm{K}$ del intestino proximal, responde a los alimentos ricos en carbohidratos y lípidos incrementando la secreción de insulina y estimulando la lipoprotien-lipasa. El PYY, al igual que el GLP-1 es secretado por las células $L$ del íleo distal, incrementa la saciedad, retrasa el vaciamiento gástrico a través del receptor del neuropéptido $Y$, e inhibe la secreción gástrica, panceática e intestinal. La grelina es una hormona peptídica secretada por las células $X$ y $A$ del fondo gástrico e intestino proximal, actúa a nivel hipotalámico incrementando el apetito, inhibe la secreción de la insulina y aumenta la expresión de enzimas que promueven el almacenamiento de grasa en el adipocito ${ }^{14}$. La CCK se secreta en el intestino delgado en el posprandio y se relaciona con la disminución en el consumo de alimento a través de receptores en el nervio vago. Por último, el PP sintetizado y secretado por el páncreas endócrino reduce el apetito en el posprandio ${ }^{15}$.

Se ha demostrado que el sistema de incretinas se encuentra alterado en los pacientes diabéticos tipo 2, aunque no se conocen exactamente todos los mecanismos, se sabe que el GIP estimula la secreción de insulina solo en un $20-30 \%$ de lo normal por un defecto en la expresión de su receptor, y por un menor número de éstos en la célula $B$ pancreática ${ }^{16}$. También se sabe que existe en los pacientes obesos y diabéticos una secreción disminuida de GLP-1, PYY y grelina.

Es posible que cambios anatómicos gastrointestinales produzcan diferentes efectos en la regulación de la glucosa. La interposición ileal, que es una cirugía en experimentación en la que se une un segmento de ileon distal con el yeyuno preservando la continuidad gastrointestinal, incrementa el GLP-1 y el PYY en roedores ${ }^{17}$ y humanos ${ }^{18}$, posiblemente por la temprana estimulación del intestino distal con alimentos parcialmente digeridos. 


\section{Diabetes como enfermedad quirúrgica}

Es ampliamente conocido que la restricción de la ingesta calórica y la pérdida de peso afectan el metabolismo de la glucosa. Los diversos tipos de cirugía bariátrica que se realizan en la actualidad nos pueden ayudar a entender esto; sin embargo, existen algunas características específicas dependiendo del tipo de procedimiento.

Mientras que los procesos restrictivos, como la banda gástrica ajustable o la gastroplastía vertical en banda, presentan porcentajes de resolución de diabetes en $45-73 \%$ en semanas o meses después del procedimiento ${ }^{19,20}$; los procesos malabsortivos como la derivación biliopancreática o combinados con procesos restrictivos como el bypass gástrico en $\mathrm{Y}$ de Roux, presentan resolución de la diabetes en días y en porcentajes de $78-99 \%{ }^{3-9,21,22,23}$, por lo que la simple pérdida de peso no puede explicar por sí solo el mejoramiento.

Para probar que no necesariamente la diabetes se remite en los pacientes obesos por la pérdida de peso asociado a la cirugía bariátrica, un estudio llevado a cabo por Pories WJ y cols, sometió a un grupo de pacientes a bypass gástrico tipo Greenville y lo comparó 24 y 30 meses después con un grupo control similar en edad, sexo, peso y duración de diabetes, descubriendo menor nivel de glucosa, insulina y leptina en aquellos sometidos a la cirugía $24,25$. Posteriormente dos estudios, uno en ratas diabéticas no obesas del tipo Goto-Kakizaki que fueron sometidas a un bypass duodeno-yeyunal con preservación del estómago ${ }^{13}$, y otro en donde se le realiza la misma cirugía a 2 seres humanos diabéticos tipo 2 no obesos ${ }^{26}$, mostraron una disminución considerable en la glucosa y hemoglobina A1c, independientemente de la pérdida de peso y/o disminución de la ingesta calórica. También se ha encontrado resolución de la diabetes en pacientes moderadamente obesos sometidos a bypass gástrico en $Y$ de Roux o derivación biliopancreática 27,28.
La derivación biliopancréatica es un tipo de cirugía en donde se hacen resecciones intestinales para disminuir la absorción de nutrientes. El bypass gástrico en $Y$ de Roux es el estándar de oro en la cirugía bariátrica y consiste en la resección del estómago dejando un reservorio conectado a una asa de yeyuno llamada "asa alimentadora". La porción distal del estómago y la porción proximal del intestino forman la "asa biliopancreática" y es anastomosada hacia una porción distal del yeyuno, a partir de donde se forma la llamada "asa común". Existen dos hipótesis para explicar el efecto de estas cirugías sobre el metabolismo de la glucosa. La primera, denominada "hipótesis del intestino distal" propuesta por Cumming y cols. ${ }^{29}$ que consiste en el incremento de GLP-1 y PYY por el contacto de nutrientes parcialmente digeridos con el intestino distal, el mismo mecanismo explicado previamente en la cirugía de interposición ileal. La segunda, llamada "hipótesis del intestino proximal", propuesta por Rubino y cols. que consiste en la prevención de la activación de los mecanismos de señalización que promueven la resistencia a la insulina en la diabetes tipo 2 al excluir al duodeno y yeyuno proximal del contacto con los alimentos 30,31 .

Para demostrar la importancia de la hipótesis del intestino proximal como el mecanismo más importante en la mejora de la homeostasis de la glucosa, Rubino y cols. realizaron un estudio que consistió en utilizar dos grupos de ratas diabéticas. El primer grupo fue sometido a un bypass duodeno yeyunal (cirugía que consiste en una gastrectomía más la exclusión del duodeno, como en el bypass gástrico en $Y$ de Roux) y el segundo grupo a una interposición ileal. Las ratas sometidas a la primera cirugía presentaron una regulación de la glucosa superior a las ratas de la segunda cirugía, misma que fue revertida al reconectar el intestino proximal previamente resecado ${ }^{32}$. 
Al contrario de lo que se esperaría, este tipo de cirugías no siempre tienen un efecto positivo en la secreción y sensibilidad a la insulina, ya que si se realizan en ratas no diabéticas existe un empeoramiento ${ }^{13}$; esto mismo coincide con los humanos no diabéticos a los que se someten a una extirpación de duodeno por patología secundaria a cáncer o ulcera péptica ${ }^{33}$. Se concluye que existe una disfunción duodeno-yeyunal exclusiva en la diabetes tipo 2 que pudiera beneficiarse de una modificación en la anatomía gastrointestinal.

\section{Teoría anti-incretinas}

Para explicar cómo la exclusión del duodeno mejora la diabetes, Rubino y cols. desarrollaron la "teoría anti-incretinas" 30 que postula la existencia de un mecanismo contrarregulador de las incretinas estimulado por el paso de nutrientes por el intestino proximal, cuya función es aumentar la resistencia a la insulina, disminuir la secreción de ésta y promover la apoptosis de la célula $B$ pancreática. Se requiere un equilibrio entre las incretinas y anti-incretinas para un nivel normal de glucosa. Si existe un desequilibrio a favor de las incretinas hay hipoglucemia, y neisidobastosis y si existe un desbalance a favor de las anti-incretinas, el desarrollo de diabetes sería evidente.

No se sabe específicamente qué factores podrían formar el sistema de anti-incretinas, pero pudieran estar interfiriendo con el receptor "defectuoso" de GIP ${ }^{34}$; se requieren más estudios al respecto.

\section{Efecto de la cirugía bariátrica en la secreción de péptidos intestinales} Se ha demostrado que los niveles de GLP-1 incrementan después del bypass gástrico en $Y$ de Roux y derivación biliopancreática ${ }^{31}$, ${ }^{35}$, no siendo así en los procedimientos restrictivos ${ }^{36}$, ni en reducciones de peso similar a través de dieta hipocalórica ${ }^{37}$. Dichos efectos se mantienen un año después del bypass gástrico en $\mathrm{Y}$ de Roux ${ }^{38}$ y hasta 20 años en un bypass yeyunoileal ${ }^{10}$.

Existe incremento del PYY posprandial desde el segundo día del bypass gástrico en $Y$ de Roux ${ }^{39}$, aunque no existen estudios que describan por cuánto tiempo permanece así. Tampoco existen suficientes estudios en procesos restrictivos, solo uno en la banda gástrica ajustable donde se refiere disminución de los niveles de este péptido ${ }^{40}$.

Existen menos estudios sobre el GIP. Laffére y cols. ${ }^{41}$ reportaron su incremento 1.6 veces durante solo 6 a 12 meses posteriores al bypass gástrico en $\mathrm{Y}$ de Roux. En contraste Clements y cols ${ }^{42}$ refieren niveles bajos de GIP desde la segunda semana de la cirugía. La explicación a esos resultados se relaciona con el bypass de las células $\mathrm{K}$ del intestino proximal y a la mejor regulación del receptor de GIP en la célula $B$ pancreática ${ }^{43}$.

Los efectos del bypass gástrico en los niveles de grelina están determinados por el tejido gástrico productor de ghrelina que se preserva posterior a la cirugía y a la integridad del nervio vago ${ }^{44}$. Los estudios reportan supresión posprandial de PP a los nueve meses en las cirugías malabsortivas mientras que no reportan cambios en los niveles de CCK ${ }^{45}$.

\section{Consideraciones especiales y limitaciones}

La evidencia anterior sostiene la posibilidad de considerar a la cirugía bariátrica no sólo como una cirugía para el tratamiento de la obesidad sino como una cirugía metabólica. Se define esta última como la modificación anatómica de regiones del tracto gastrointestinal para el mejoramiento de la diabetes a través de distintos mecanismos fisiológicos.

Se debe de considerar que como cualquier procedimiento quirúrgico, una cirugía metabólica para el tratamiento de 
la diabetes conlleva el riesgo de muerte y complicaciones pos-quirúrgicas.

Los procedimientos quirúrgicos metabólicos no producen el mismo control glucémico en pacientes con diabetes tipo $1 \mathrm{y}$ diabéticos tipo 2 de larga evolución (8-10 años) debido a la reserva de células $B$ escasa o nula ${ }^{46}$. La medición de péptido- $C$, anticuerpos anti-islote y reserva de célula $B$ pancreática deberán incluirse en el futuro como indicadores de selección para los candidatos a cirugía. Otros factores pronósticos pre y posoperatorios que afectan la resolución de la diabetes son: el pobre control glucémico, el uso previo de insulina y la circunferencia de la cintura ${ }^{22}$. Se requiere continuar investigando otro tipo de opciones terapéuticas para ese tipo de pacientes.

Aunque con resultados prometedores, no existen suficientes estudios de cirugía bariátrica en pacientes con $\mathrm{IMC}<35 \mathrm{~kg} / \mathrm{m}^{2}$.
La poca evidencia en humanos y animales no demuestra cambio significativos en la curva ponderal en pacientes no obesos 26,33,47; sin embargo, quizá sea preferible realizar una cirugía como un bypass duodeno yeyunal con preservación de fondo gástrico que ha demostrado presentar menos cambios en peso pero si mejorar el control glucémico.

Pacientes que serían candidatos a cirugía metabólica incluiría a aquellos con edad entre 20 y 65 años, con índice de masa corporal (IMC) entre $23-34 \mathrm{~kg} / \mathrm{m}^{2}$, con evolución menor de 10 años de la diabetes, en tratamiento previo con antidiabéticos orales o insulina (menos de 7 años), con control inadecuado de la diabetes (hemoglobina $A 1 c>7.5 \%$ ), y una comprensión adecuada de los mecanismos de la cirugía por parte del paciente.

\section{Conclusiones}

Las cirugías gastrointestinales convencionales para el tratamiento de la obesidad mórbida mejoran considerablemente la diabetes tipo 2, permitiendo una normalización de los niveles de glucosa, hemoglobina A1c y suspensión de medicamentos. Estos cambios surgen antes de la pérdida de peso, sugiriendo que existen otros mecanismos producto de la modificación de la anatomía intestinal que ayudan al mejor control de la diabetes. Existe cada vez más evidencia del importante papel de los mediadores hormonales producidos a nivel intestinal en la fisiopatología de la diabetes. A través de mecanismos no muy establecidos se sabe que GLP-1 PYY y grelina se encuentran disminuidos en pacientes diabéticos mientras que los niveles de GIP incrementados. Los procedimientos quirúrgicos en donde se excluye el contacto del alimento con el duodeno y yeyuno proximal y se exponen parcialmente digeridos directamente con el intestino distal incrementan los niveles de GLP-1 y PYY restableciendo el equilibrio previamente perdido jugando efectivamente un rol en el tratamiento de la diabetes tipo 2. Se tiene que determinar si el efecto quirúrgico además de mejorar el metabolismo de la glucosa es capaz de revertir anormalidades presentados con anterioridad. Sin embargo, es necesario contar con mayor evidencia nivel 1 que pruebe la eficacia de los procedimientos antes descritos así como de los nuevos procedimientos que se describen bajo protocolos aprobados a corto y largo plazo. 


\section{Bibliografía}

1 Wild S. Roglic G. Green A. et al. Global Prevalence of Diabetes. Estimates for the year 2000 and projections for 2030. Diabetes Care 2004; 27:104753.

2 Arredondo A. Zúñiga A. Economic consecuences of epidemiological changes in diabetes in middle income countries: the mexican case, Diabetes Care 2004; 27: 104-9.

3 Sjostrom L. Lindroos AK. Peltonen $M$, et al. Lifestyle, diabetes, and cardiovascular risk factors 10 years after bariatric surgery. N Engl J Med 2004; 351:2683-93.

4 Eric J. DeMaria. Bariatric surgery for morbid obesity. N Engl J Med 2007; 356:2176-83.

5 Romero Ibargüengoitia $M E$, Lerman I, Herrera MF et al. Cirugía bariátrica y obesidad mórbida. Experiencia en el Instituto Nacional de Ciencias Médicas y Nutrición Salvador Zubirán. 2009 RIC en prensa.

6 Buchwald H. Avidor Y. Braunwald E, et al. Bariatric surgery a systematic review and meta-analisis. JAMA 2004; 292:1724-37.

7 Adams TD. Gress RE. Smith SC, et al. Long Term Mortality after bypass surgery. N.Engl J Med 2007; 257: 735-61.

8 Maggard MA. Shugarman LR. Suttorp M, et al Meta-analisis: Surgical treatment of obesity. Ann Intern Med 2005; 142:547-59.

9 Pories WJ. Swansons. MacDonald KG, et al. Who would have thought it? An operation proves to be the most effective therapy for adult-onset diabetes mellitus. Ann Surg 1995; 222:339-350.

10 Marion L. Vetter. Michael R. Nayyar Iqbal. Narrative Review: Effect of Bariatric Surgery on Type 2 Diabetes Mellitus. Ann Intern Med 2009; 150: $94-$ 103.

11 Fetner R, McGinty J, Russell C, Pi-Sunyer FX, Laferrère $B$. Incretins, diabetes and bariatric surgery: a review. Surg Obes Relat Dis. 2005; 1:589-97.

12 Toft-Nielsen MB. Madsbad S. Holst JJ. Continuous subcutaneus infusion of glucagon like peptid 1 lowers plasma glucose and reduces appetite in type 2 diabetes patients. Diabetes Care 1999; 22: $1137-$ 43.

13 Hansoria T. Drucker DJ. GIP and GLP-1 as incretin hormons lessons from single and double incretin receptor knockout mice. Regul Pept. 2005; 128: 125 34
14 Cummings DE. Shannon MH. Ghrelin and gastric bypass: is there a hormonal contribution to surgical weight loss? J Clin Endocrnol Metab 2003; 88: 2999-3002.

15 Kevin G. Murphy\&Stephen R. Bloom. Gut hormones and the regulation of energy homeostasis. $\mathrm{Na}$ ture 2006; 44;854-9.

16 Holst JJ. Gromada J. Nauck MA. The patogénesis of NIDDM involves a defective expresión of the GIP receptor. Diabetologia 1997; 40:984-6.

17 Strader AD. Vahl TP. Jandacek RJ. Woods SC D'Alessio DA. Seeley RJ. Weight loss through ileal transposition is accompanied by increased ileal hormone secretion and synthesis in rats. Am J Physio Endocrinol Metab 2005; 288: 447-453.

18 Mason EE. Ileal [correction of ilial] transposition and enteroglucagon/GLP-1 in obesity (and diabetic?) surgery. Obes Surg. 1999; 9:223-228.

19 Pontiroli AE. Pizzocri P. Librenti MC, et al. Laparsocopic adjustable gastric banding for the treatment of morbid obesity and its metabolic complications: a three-year study. J. Clin Endocrinol Metab 2002; 8: $3555-61$

20 Dixon JB. O'Brien PE. Playfair J, et al. Adjustable gastric banding and conventional therapy for type 2 diabetes a randomized controlled trial. JAMA 2008; 299: 316-23

21 Scopinaro N, Marinari GM, Camerini GB, Papadia FS, Adami GF. Specific effects of biliopancreatic diversion on the mayor components of metabolic syndrome: a long term follow up study. Diabetes Care 2005; 28: 2406-11

22 Schauer PR. Burguera B. Ikramuddin S. Effect of laparoscopic roux en y gastric bypass on type 2 diabetes mellitus. Ann Surg 2003; 238:46-84.

23 Buchwald H. Estok R. Fahrback K, et al Weight and type 2 diabetes after bariatric surgery: systematic review and meta-analysis. Am J Med 2009; 122:248-256.

24 Hickey Ms. Pories WJ. Mac Donald KGJr, Et al. A new paradigm for type 2 diabetes mellitus: could it be a disease of the foregut? Ann Surg 1998; 227: $637-43$

25 Pories WJ. Swanson Ms. Mad Donald Kg, et al Who would have thought it? An oeration proves to be the most effective therapy for adult-onset diabetes Mellitus. Ann Surg 1995; 222:3399-50 
26 Cohen RV. Schiavon CA. Pinheiro JS. Correa JL. Rubino F. Duodenal-jejunal bypass for the treatment of type 2 diabetes in patients with body mass index of $22-34 \mathrm{~kg} / \mathrm{m} 2$ : a report of 2 cases. Surg Obes Relat Dis 2007 3:195-7.

27 Noya G, Cossu ML. Coppola M, et al. Biliopancreatic diversion preserving the stomach and pylorus in the treatment of hypercholesterolemia and diabetes type II: results in the first 10 cases Obes Surg 1998; 8: 67-72.

28 Cohen R. Pinheiro JS. Correa JL. Schiavon CA. Laparoscopic Roux-en-Y gastric bypass for BMI $<35$ $\mathrm{kg} / \mathrm{m}(2)$ : a tailored approach. Surg Obes Relat Dis 2006; 2:401-404.

29 Cummings DE. Overduin J. Foster-Schubert KE. Carlson MJ. Role of the bypassed proximal intestine in the anti-diabetic effecs of bariatric surgery. Surg Obes Relat 2007; 3:109-15.

30 Rubino F. Is type 2 diabetes an operable intestinal disease? A provocative yet reasonable hypotesis. Diabetes Care. 2008; 2: 290-6.

31 Mason EE. The mechanism of surgical treatment of type 2 diabetes. Obes Surg 2005; 15: 459-61.

32 Rubino F. Forgione A. Cummings DE. The mechanism of diabetes control after gastrointestinal bypass surgery reveals a role of the proximal small intestine in the pathophysiology of type 2 diabetes. Ann Surg 2006; 244: 741-49.

33 Schwarz A. Büchler M. Usinger K. Importance of the duodenal passage and pouch volume after total gastrectomy and reconstruction with the ulm pouch: prospective randomized clinical study. World J Surg 1996; 20: 60-67.

34 Lynn FC. Pamir N. Ng EH. McIntosh CH. Kieffer TJ. Pederson RA. Defective glucose-dependent insulinotropic polypeptide receptor expression in diabetic fatty Zucker rats. Diabetes 2001; 50: 1004-11.

35 Cohen RV. Schiavon CA. Pinheiro JS. Correa JL. Rubino F. Duodenal-jejunal bypass for the tretment of type 2 diabetes in patients with body mass index $22-34 \mathrm{~kg} / \mathrm{m} 2$ a report of 2 cases. Surg Obes Relat Dis. 2007; 2:195-7.

36 Rodieux F. Giusti V.D'Alessio DA. Suter M. Tappy L. Effects of gastric bypass and gastric banding on glucose kinetics and gut hormone release. Obesity 2008; 16:298-305.
37 Laferrère B. Teixeira J. McGinty J, et al. Effect of weight loss by gastric bypass surgery versus hypocaloric diet on glucose and incretin levels in patients with type 2 diabetes. J Clin Endocrinol Metab. 2008; 93: 2479-85.

38 Laferrére B. Tran $\mathrm{H}$. Egger JR, et al. The increase in GLP-1 levels and incretin effect after Roux en $Y$ gastric bypass surgery (RYGBP) persists up to 1 year in patients with type 2 diabetes (T2DM) Obesity 2007; $15: 7$

39 le Roux CW. Welbourn R. Werling $M$, et al. Gut hormones as mediators of appetite and weight loss after Roux en $\mathrm{Y}$ gastric bypass. Ann Surg 2007; 246: 780-5.

40 Korner J. Inabnet W. Conwell IM, et al. Differential effects of gastric bypass and banding on circulating gut hormone and leptin levels. Obesity 2006; 14: 1553-61.

41 Laferrère B. Heshka S. Wang K, et al. Incretin levels and effect are markedly enchanced 1 month after Roux en $Y$ gastric bypass sugery in obese patients with type 2 diabetes. Diabetes Care 2007; 30:1709-16.

42 Clements RH. Gonzalez QH. Long Cl. Wittert G. Laws HL. Hormonal changes after Roux- en $Y$ gastric bypass for morbid obesity and the control of type-II diabetes mellitus. Am Surg 2004; 70: 1-4.

43 Patriti A. Facchiano E. Sanna A. Gullà N. Donini A. The enteroinsular axis and the recovery frome type 2 diabetes after bariatric surgery. Obes Surg 2004; 14:840.

44 Cummings DE. Weigle DS. Frayo RS et al. Ghrelin: a gut-brain hormone: effects on gastric bypass surgery. Obes Surg 2003; 88: 3177-88.

45 Vincent RP, Le Roux CW. Changes in Gut Hormones after bariatric Surgery.Clin Endocrinol 2008; 69: 173-179.

46 Cossu ML. Noya G. Tonolo GC, et al. Duodenal switch without gastric resection: results and observations after 6 years. Obes Surg 2004; 14:1354-9.

47 Ramos AC. Galvão Neto MP. de Souza YM, et al. Laparoscopic Duodenal-Jejunal Exclusion in the Treatment of Type 2 Diabetes Mellitus in Patients with $\mathrm{BMI}<30 \mathrm{~kg} / \mathrm{m}(2)$ (LBMI). Obes Surg. 2008 Nov 6. [Epub ahead of print] 